\author{
Research Article
}

Jesus Ildefonso Díaz*, David Gómez-Castro, Alexander V. Podol’skii and Tatiana

A. Shaposhnikova

\title{
Characterizing the strange term in critical size homogenization: Quasilinear equations with a general microscopic boundary condition
}

https://doi.org/10.1515/anona-2017-0140

Received June 14, 2017; accepted June 16, 2017

\begin{abstract}
The aim of this paper is to consider the asymptotic behavior of boundary value problems in $n$ dimensional domains with periodically placed particles, with a general microscopic boundary condition on the particles and a $p$-Laplace diffusion operator on the interior, in the case in which the particles are of critical size. We consider the cases in which $1<p<n, n \geq 3$. In fact, in contrast to previous results in the literature, we formulate the microscopic boundary condition in terms of a Robin type condition, involving a general maximal monotone graph, which also includes the case of microscopic Dirichlet boundary conditions. In this way we unify the treatment of apparently different formulations, which before were considered separately. We characterize the so called "strange term" in the homogenized problem for the case in which the particles are balls of critical size. Moreover, by studying an application in Chemical Engineering, we show that the critically sized particles lead to a more effective homogeneous reaction than noncritically sized particles.
\end{abstract}

Keywords: Homogenization, $p$-Laplace diffusion, nonlinear boundary reaction, noncritical sizes, maximal monotone graphs

MSC 2010: 35B25, 35B40, 35J05, 35J20

\section{Introduction}

A well-known effect in homogenization theory is the appearance of some changes in the structural modelling of the homogenized problem for suitable critical size of the elements configuring the "micro-structured" material. It seems that the first result in this direction was presented in the pioneering paper by Marchenko and Hruslov [27]. A more popular presentation of the appearance of some "strange terms" was due to Cioranescu and Murat [4]. Both articles dealt with linear equations with Neumann and Dirichlet boundary conditions, respectively. Since then many papers were devoted to different formulations, e.g., more general elliptic partial differential equations (possibly of quasilinear type), Robin type and other boundary conditions of different nature, etc. It is impossible to mention all of them here (a few of them will be mentioned in the rest of the introduction) but the reader may imagine that the nature of this "strange term" may be completely different according to the peculiarities of the formulation in consideration (something that was already indicated at the end of the introduction of the paper by Cioranescu and Murat [4]).

\footnotetext{
*Corresponding author: Jesus Ildefonso Díaz, Departamento de Matemática Aplicada e I.M.I., Universidad Complutense de Madrid, Plaza de las Ciencias 3, 28040 Madrid, Spain, e-mail: jidiaz@ucm.es. https://orcid.org/0000-0003-1730-9509 David Gómez-Castro, Departamento de Matemática Aplicada e I.M.I., Universidad Complutense de Madrid, Plaza de las Ciencias 3, 28040 Madrid, Spain, e-mail: dgcastro@ucm.es

Alexander V. Podol'skii, Tatiana A. Shaposhnikova, Faculty of Mechanics and Mathematics, Moscow State University, Moscow 199992, Russia, e-mail: originalea@ya.ru, shaposh.tan@mail.ru 
The main goal of this paper is to characterize the change of structural behavior arising in the homogenization process when applied to chemical reactions taking place on fixed-bed nanoreactors, at the microscopic level, on the boundary of the particles

$$
\begin{cases}-\Delta_{p} u_{\varepsilon}=f(x), & x \in \Omega_{\varepsilon}, \\ -\partial_{v_{p}} u_{\varepsilon} \in \varepsilon^{-\gamma} \sigma\left(u_{\varepsilon}\right), & x \in S_{\varepsilon}, \\ u_{\varepsilon}=0, & x \in \partial \Omega\end{cases}
$$

for a very general type of chemical kinetics (here given by the maximal monotone graph $\sigma$ of $\mathbb{R}^{2}$ ). Thanks to this generality on the maximal monotone graph $\sigma$, our treatment also includes the case of microscopic Dirichlet boundary conditions. In this way we unify the treatment of apparently different formulations, which before were considered separately.

The diffusion is modeled by the quasilinear operator $\Delta_{p} u_{\varepsilon} \equiv \operatorname{div}\left(\left|\nabla u_{\varepsilon}\right|^{p-2} \nabla u_{\varepsilon}\right)$, with $p>1$. Notice that $p=2$ corresponds to the linear diffusion operator, and that $p \neq 2$ appears in turbulent regime flows or nonNewtonian flows (see [8]). As it is well known, this operator appears in many other contexts and is one of the best examples of quasilinear operators leading to a formulation in terms of nonlinear monotone operators (see, e.g., $[1,7,26])$.

The "normal derivative" must be then understood as $\partial_{v_{p}} u_{\varepsilon}=\left|\nabla u_{\varepsilon}\right|^{p-2} \nabla u_{\varepsilon} \cdot v$, where $\boldsymbol{v}$ is the outward unit normal vector on the boundary of the particles $S_{\varepsilon} \subset \partial \Omega_{\varepsilon}$. In fact, we will consider the structural assumption

$$
1<p<n, \quad n \geq 3 .
$$

The cases $p \geq n$ are completely different, see [31, 32] (see also, for instance, the study made for a general monotone quasilinear equation with Dirichlet boundary conditions in [7]).

As mentioned before, the generality assumed on the maximal monotone graph $\sigma$ of $\mathbb{R}^{2}$ allows to treat, in a unified way, different cases as the case of Dirichlet boundary conditions, which corresponds to the choice of $\sigma$ given by

$$
D(\sigma)=\{0\} \quad \text { and } \quad \sigma(0)=(-\infty,+\infty)
$$

(see, e.g. [1]), and the case of nonlinear Robin type boundary conditions, which corresponds (see, e.g. [24]) to the case in which $D(\sigma)=\mathbb{R}$ and $\sigma$ is a continuous nondecreasing function.

The domain $\Omega_{\varepsilon} \subset \mathbb{R}^{n}$ is assumed to have an $\varepsilon$-periodical structure. Since our main goal is to get a very precise description of the so-called "strange term" in the homogenized problem, we shall assume that the particles are balls of radius $a_{\varepsilon}=C_{0} \varepsilon^{\alpha}$, where $\alpha>1$. One of the interesting properties that arise from our precise characterization is that there is uniqueness of solutions of the homogenized problem. This was not always proved in previous results (cf. the general framework considered in [7], and how their characterization, given in their Lemma 5.1, is not enough to get the uniqueness of solution of their homogenized problem). The consideration of particles of a general shape is a difficult task, especially the exact identification of the "strange terms". A similar formulation to the one considered in this paper for that case can be obtained, at least for continuous $\sigma$, and has been the subject of a different paper (see [15]).

The problem has two different parameters: $\alpha$, the size of the particles, and $\gamma$, the normalization factor of the boundary condition on $S_{\varepsilon}$. When they have critical values

$$
\alpha=\frac{n}{n-p}, \quad \gamma=\alpha(n-1)-n=\alpha(p-1),
$$

then our main result in this paper shows that the homogenized problem involves a different distributed chemical kinetics nonlinearity:

$$
\begin{cases}-\Delta_{p} u+\mathcal{A}|H(u)|^{p-2} H(u)=f(x) & \text { in } \Omega, \\ u=0 & \text { on } \partial \Omega,\end{cases}
$$

where

$$
\mathcal{A}=\left(\frac{n-p}{p-1}\right)^{p-1} C_{0}^{n-p} \omega_{n}
$$


and $H: \mathbb{R} \rightarrow \mathbb{R}$ is given by

$$
H(r)=\left(I+\sigma^{-1} \circ \Theta_{n, p}\right)^{-1}(r)
$$

with

$$
\Theta_{n, p}(s)=\mathcal{B}_{0}|s|^{p-2} s \quad \text { for } s \in \mathbb{R}
$$

and

$$
\mathcal{B}_{0}=\left(\frac{n-p}{C_{0}(p-1)}\right)^{p-1},
$$

where $\omega_{n}$ is the surface area of the unit sphere in $\mathbb{R}^{n}$. We show that, for any maximal monotone graph $\sigma, H$ is a nondecreasing contraction, and thus the existence, uniqueness and continuous dependence of solutions of the homogenized problem is consequence of well-known results on monotone operator theory.

The change of behavior from the nonlinearity of type $\sigma$ in the nonhomogeneous problem to the nonlinearity $H$ in the homogeneous problem is one of the characteristics of the nanotechnological effects (see, e.g., [33]) and does not appear if $1 \leq \alpha<\frac{n}{n-p}$ (see [5, 34]).

Before presenting the details of the notation used above, let us mention that our main aim is to provide a common roof and extend (under different points of view) some previous results in the literature concerning different structural assumptions (i.e., the functions $\sigma$ and $H$ ) after the homogenization process.

The case of Robin boundary conditions $\partial_{n} u+\beta(\varepsilon) \sigma(u)=0$ on $S_{\varepsilon}$ was first studied by Marchenko and Hruslov in a series of papers dealing mainly with the linear case $\sigma(u)=\lambda u$, see [19-21, 27]. Some references on different choices of smooth functions $\sigma$ can be found in $[6,18,22,24,25,29,37]$ and the references therein. For further references, see $[13,16,17,23]$. Some previous results by the authors [11], formulated there for some not necessarily Lipschitz functions $\sigma$ and $p \in[2, n)$, will be here extended to the case a general maximal monotone graph $\sigma$ (which includes the case of Dirichlet boundary conditions) and $p \in(1, n)$.

The special case of Dirichlet boundary condition $u_{\varepsilon}=0$ on $S_{\varepsilon}$, covered by (1.2), gives $\sigma^{-1}(s)=0$ for any $s \in \mathbb{R}$, and so $H(r)=r$ for any $r \in \mathbb{R}$. Therefore, the "strange term" arising in the homogenized equation becomes $\mathcal{A}|u|^{p-2} u$. This was shown for $p=2$ in the pioneering paper by Cioranescu and Murat [4]. However, even in this simple case, the treatment in [7] for the case $p \neq 2$ is not as sharp as in our case. In [7], Dal Maso and Skrypnik do not provide an explicit expression for this strange term. In fact, their characterization (see [7, Lemma 5.1]) does not guaranty uniqueness of solutions of the homogenized problem.

The case of the boundary condition

$$
u_{\varepsilon} \geq 0, \quad \partial_{n} u_{\varepsilon}+\varepsilon^{-\gamma} \sigma_{0}\left(u_{\varepsilon}\right) \geq 0, \quad u_{\varepsilon}\left(\partial_{n} u_{\varepsilon}+\varepsilon^{-\gamma} \sigma_{0}\left(u_{\varepsilon}\right)\right)=0 \quad \text { on } S_{\varepsilon},
$$

which was studied for smooth $\sigma_{0}$ in [22] by ad hoc techniques, is also covered by the common proof provided in this paper, by taking

$$
D(\sigma)=[0,+\infty), \quad \sigma(u)= \begin{cases}(-\infty, 0] & \text { if } u=0 \\ \sigma_{0}(u) & \text { if } u>0\end{cases}
$$

See also [12, 28].

The choice of the critical values of $\alpha$ and $\gamma$ might appear arbitrary. Let us give some reasons why this is a good choice. First, if $N(\varepsilon)$ is the number of particles, then $N(\varepsilon) \sim \varepsilon^{-n}$. It is easy to see that $\left|S_{\varepsilon}\right|=$ $N(\varepsilon)\left|\partial\left(a_{\varepsilon} G_{0}\right)\right| \sim \varepsilon^{\alpha(n-1)-n}$, where $G_{0}$ is the unit ball centered at 0 . Let us analyze the choice of $\gamma$. If we consider the reaction term on the weak formulation, with $\sigma\left(u_{\varepsilon}\right)$ a bounded sequence in $L^{\infty}$ and $v$ a bounded test function, then

$$
\frac{1}{\left|S_{\varepsilon}\right|} \int_{S_{\varepsilon}} \sigma\left(u_{\varepsilon}\right) v d S \sim \varepsilon^{-(\alpha(n-1)-n)} \int_{S_{\varepsilon}} \sigma\left(u_{\varepsilon}\right) v d S
$$

is a bounded sequence. Hence, if the sequence $u_{\varepsilon}$ is bounded in $L^{\infty}$ and $v$ is a bounded test function, then

$$
\varepsilon^{-\gamma} \int_{S_{\varepsilon}} \sigma\left(u_{\varepsilon}\right) v d S
$$

can only be expect to tend to either 0 or $+\infty$ if $\gamma \neq \alpha(n-1)-n$, and hence we will lose the reaction term on the equation on the homogenized equation or we lose the equation altogether. If the macroscopic behavior 
is given by a reaction diffusion equation (with nontrivial reaction), then the choice scaling $y$ as $\varepsilon \rightarrow 0$ can be no other.

The appearance of the critical value of $\alpha$ has to do with a property of traces. It is known (see [30]) that

$$
\int_{a_{\varepsilon} S_{0}}|u|^{p} d S \leq K\left(a_{\varepsilon}^{n-1} \varepsilon^{-n} \int_{Y_{\varepsilon}}|u|^{p} d x+a_{\varepsilon}^{p-1} \int_{Y_{\varepsilon}}|\nabla u|^{p} d x\right) .
$$

As it turns out, the critical scale is the one in which both terms in the right-hand side have the same order of convergence. Notice that, in the critical case $\alpha=\frac{n}{n-p}$, we have $\gamma=\alpha(p-1)$.

Notice that for a Newtonian fluid in $\mathbb{R}^{3}(n=3, p=2)$, the critical size corresponds to $\alpha=3$. Obviously the critical value of $\alpha$ is an increasing function of $p$. Therefore, for non-Newtonian dilatant fluids or a Newtonian flow in turbulent regime ( $p>2$ ), our assumption means $\alpha>3$, the particles are tiny with respect to their repetition, whereas for pseudoplastic fluids ( $p<2$ ), the critical particles satisfy $\alpha<3$, and hence are not so tiny with respect to their repetition.

A relevant application of our results is the following. Let us consider the usual formulation in Chemical Engineering (see $[9,35])$ with a constant external supply

$$
\begin{cases}-\Delta w_{\varepsilon}=0, & x \in \Omega_{\varepsilon}, \\ \partial_{\nu} w_{\varepsilon}+\varepsilon^{-\gamma} g\left(w_{\varepsilon}\right)=0, & x \in S_{\varepsilon}, \\ w_{\varepsilon}=1, & x \in \partial \Omega,\end{cases}
$$

where $g$ is a nondecreasing real function such that $g(0)=0$. In order to adapt our results, we introduce the change in variable $u=1-w$ and $\sigma(u)=g(1)-g(1-u)$, and the problem becomes

$$
\begin{cases}-\Delta u_{\varepsilon}=0, & x \in \Omega_{\varepsilon}, \\ \partial_{\nu} u_{\varepsilon}+\varepsilon^{-\gamma} \sigma\left(u_{\varepsilon}\right)=\varepsilon^{-\gamma} g(1), & x \in S_{\varepsilon}, \\ u_{\varepsilon}=0, & x \in \partial \Omega .\end{cases}
$$

Notice that the presence of $w_{\varepsilon}=1$ on $\partial \Omega$ is translated to a source in $S_{\varepsilon}$ for $u_{\varepsilon}$. We will see later (Theorem 6.2) that the new equation for $H$, when $\alpha=\frac{n}{n-2}$, is

$$
\frac{n-2}{C_{0}} H(s)=\sigma(s-H(s))-g(1),
$$

that is,

$$
H(u)=-\left(g^{-1}\left(\frac{n-2}{C_{0}} \cdot\right)+\mathrm{Id}\right)^{-1}(1-u),
$$

so that an extension of $w_{\varepsilon}$ converges weakly in $H^{1}(\Omega)$ to $w_{\text {crit }}$, the solution of

$$
\begin{cases}-\Delta w_{\text {crit }}+\mathcal{A} h\left(w_{\text {crit }}\right)=0 & \text { in } \Omega, \\ w_{\text {crit }}=1 & \text { on } \partial \Omega,\end{cases}
$$

and $h$ is given by

$$
h(w)=\left(g^{-1}\left(\frac{n-2}{n} \cdot\right)+\mathrm{Id}\right)^{-1}(w) .
$$

Notice that in the case of Neumann problems, $\sigma(s) \equiv 0$ for any $s \in R$, and although $\sigma^{-1}$ is a well-known maximal monotone graph, the more direct identification of the "strange term" $H(u)$ is obtained trough the implicit equation (1.7), since in this case we get that

$$
H(s)=-\frac{C_{0} g(1)}{n-2} \quad \text { for any } s \in \mathbb{R} .
$$

In the noncritical cases, $1<\alpha<\frac{n}{n-2}$, we will show that an extension of $w_{\varepsilon}$ converges weakly in $H^{1}(\Omega)$ to $w_{\text {non-crit }}$, the solution of

$$
\begin{cases}-\Delta w_{\text {non-crit }}+\hat{\mathcal{A}} g\left(w_{\text {non-crit }}\right)=0 & \text { in } \Omega, \\ w_{\text {non-crit }}=1 & \text { on } \partial \Omega,\end{cases}
$$


with $\hat{\mathcal{A}}=C_{0}^{n-1}\left|\partial G_{0}\right|$. Finally, we will show in Theorem 6.3 that

$$
w_{\text {crit }} \geq w_{\text {non-crit }}
$$

so we have a pointwise "better" reaction in the critical case [10]. We point out that a different criterion to establish the optimality of the reaction in terms of the so-called "chemical effectiveness" was considered by the authors in [14].

The plan of the rest of the paper is the following: Section 2 will be devoted to the statement of the main results, Section 3 contains the proof of the existence results for equation (1.1) and the characterization of $H$, Section 4 is devoted to the proof of the main result, Theorem 2.4, and Section 5 contains the proof of the auxiliary Theorem 2.9, which studies the limit of the diffusion. We conclude the paper with Section 6, where we study the noncritical case and the pointwise comparison of its homogenized solution with the critical case.

\section{Statement of the main results}

Let $\Omega$ be a bounded domain in $\mathbb{R}^{n}, n \geq 3$, with a smooth boundary $\partial \Omega$, and let $Y=\left(-\frac{1}{2}, \frac{1}{2}\right)^{n}$. Denote by $G_{0}=B_{1}(0)$ the unit ball centered at the origin. This plays a crucial role in the proof. As far as we known, no results are known in the critical cases if $G_{0}$ is not a ball. For $\delta>0$ and $\varepsilon>0$, we define sets $\delta B=\left\{x: \delta^{-1} \chi \in B\right\}$ and $\widetilde{\Omega}_{\varepsilon}=\{x \in \Omega: \rho(x, \partial \Omega)>2 \varepsilon\}$. Let

$$
a_{\varepsilon}=C_{0} \varepsilon^{\alpha},
$$

where $\alpha>1$ and $C_{0}$ is a given positive number. Define

$$
G_{\varepsilon}=\bigcup_{j \in \Upsilon_{\varepsilon}}\left(a_{\varepsilon} G_{0}+\varepsilon j\right)=\bigcup_{j \in \Upsilon_{\varepsilon}} G_{\varepsilon}^{j},
$$

where $\Upsilon_{\varepsilon}=\left\{j \in \mathbb{Z}^{n}:\left(a_{\varepsilon} G_{0}+\varepsilon j\right) \cap \overline{\widetilde{\Omega}}_{\varepsilon} \neq \emptyset\right\}, N(\varepsilon)=\left|\Upsilon_{\varepsilon}\right| \cong \varepsilon^{-n}$, and $\mathbb{Z}^{n}$ denotes the set of vectors $z$ with integer coordinates. Define $Y_{\varepsilon}^{j}=\varepsilon Y+\varepsilon j$, where $j \in \Upsilon_{\varepsilon}$ and note that $\bar{G}_{\varepsilon}^{j} \subset \bar{Y}_{\varepsilon}^{j}$ and center of the ball $G_{\varepsilon}^{j}$ coincides with the center of the cube $Y_{\varepsilon}^{j}$. Our "microscopic domain" is defined as

$$
\Omega_{\varepsilon}=\Omega \backslash \overline{G_{\varepsilon}}, \quad S_{\varepsilon}=\partial G_{\varepsilon}, \quad \partial \Omega_{\varepsilon}=\partial \Omega \cup S_{\varepsilon} .
$$

We define the space $W_{0}^{1, p}\left(\Omega_{\varepsilon}, \partial \Omega\right)$ as the completion, with respect to the norm of $W^{1, p}\left(\Omega_{\varepsilon}\right)$, of the set of infinitely differentiable functions in $\bar{\Omega}_{\varepsilon}$ equal to zero in a neighborhood of $\partial \Omega$, that is,

$$
W_{0}^{1, p}\left(\Omega_{\varepsilon}, \partial \Omega\right)=\left\{u \in W^{1, p}\left(\Omega_{\varepsilon}\right): u=0 \text { on } \partial \Omega\right\} .
$$

Concerning the solvability of problem (1.1), we start by introducing the notion of weak solution. Since we assume that $\sigma: \mathbb{R} \rightarrow \mathcal{P}(\mathbb{R})$, where $\mathcal{P}(\mathbb{R})$ denotes the set of subsets of $\mathbb{R}$, we recall, by well-known results (see, e.g., [2]), that

$$
\sigma \text { is a maximal monotone graph of } \mathbb{R}^{2}, 0 \in \sigma(0),
$$

and that there exists a convex lower semicontinuous function $\Psi: \mathbb{R} \rightarrow(-\infty,+\infty]$, with $\Psi(0)=0$, such that $\sigma=\partial \Psi$ is its subdifferential. We also know that if we define

$$
D(\sigma)=\{r \in \mathbb{R} \text { such that } \sigma(r) \neq \emptyset\},
$$

where $\emptyset$ denotes the empty set, and

$$
D(\Psi)=\{r \in \mathbb{R} \text { such that } \Psi(r)<+\infty\},
$$

then $D(\sigma) \subset D(\Psi) \subset \overline{D(\Psi)}=\overline{D(\sigma)}$.

In the rest of the paper we will always assume that $f \in L^{p^{\prime}}(\Omega)$, where, as usual, $p^{\prime}=\frac{p}{p-1}$. 
Since $u_{\varepsilon}$ is the minimizer of the following energy functional in $W^{1, p}\left(\Omega_{\varepsilon}, \partial \Omega\right)($ see $[1,26])$ :

$$
E(u)=\int_{\Omega_{\varepsilon}}|\nabla u|^{p} d x+\varepsilon^{-\gamma} \int_{S_{\varepsilon}} \Psi(u) d S-\int_{\Omega_{\varepsilon}} f u d x,
$$

we consider the following definition of weak solution.

Definition 2.1. We will say that $u_{\varepsilon} \in W^{1, p}\left(\Omega_{\varepsilon}, \partial \Omega\right)$ is a weak solution of problem (1.1) if $u_{\varepsilon}(x) \in D(\Psi)$ for a.e. $x \in S_{\varepsilon}$, and for all $v \in W^{1, p}\left(\Omega_{\varepsilon}, \partial \Omega\right)$, we have

$$
\int_{\Omega_{\varepsilon}}\left|\nabla u_{\varepsilon}\right|^{p-2} \nabla u_{\varepsilon} \cdot \nabla\left(v-u_{\varepsilon}\right) d x+\varepsilon^{-\gamma} \int_{S_{\varepsilon}}\left(\Psi(v)-\Psi\left(u_{\varepsilon}\right)\right) d S \geq \int_{\Omega_{\varepsilon}} f\left(v-u_{\varepsilon}\right) d x .
$$

The existence and uniqueness of a weak solution to problem (2.2) is an easy consequence of well-known results:

Proposition 2.2. There exists a unique $u_{\varepsilon} \in W^{1, p}\left(\Omega_{\varepsilon}, \partial \Omega\right)$ weak solution of (2.2). Besides, there exists $K>0$ independent of $\varepsilon$ such that

$$
\left\|\nabla u_{\varepsilon}\right\|_{L^{p}\left(\Omega_{\varepsilon}\right)}+\varepsilon^{-\gamma}\left\|\Psi\left(u_{\varepsilon}\right)\right\|_{L^{1}\left(S_{\varepsilon}\right)} \leq K
$$

The homogenized problem will involve the function $H: \mathbb{R} \rightarrow \mathbb{R}$ given by (1.5). Let us present some of the properties satisfied by $H$.

Lemma 2.3. If $\sigma$ satisfies (2.1), then the function $H$ defined by (1.5) is a nondecreasing nonexpansion on $\mathbb{R}$ (i.e., a nondecreasing Lipschitz continuous function of Lipschitz constant $L \leq 1$ ). Moreover, this function $H$ is the unique function $H: \mathbb{R} \rightarrow \mathbb{R}$ satisfying the relation

$$
\mathcal{B}_{0}|H(r)|^{p-2} H(r) \in \sigma(r-H(r)) \quad \text { for any } r \in \mathbb{R} .
$$

Concerning the homogenized problem (1.3), we point out that since $H$ is a nondecreasing nonexpansion on $\mathbb{R}$, for the parameters $\mathcal{A}$ and $\mathcal{B}_{0}$ given by (1.4) and (1.6), and for $f \in L^{p^{\prime}}(\Omega)$, there exists a unique weak solution $u \in W_{0}^{1, p}(\Omega)$ of problem (1.3). Moreover, $|H(u)|^{p-2} H(u) \in L^{p^{\prime}}(\Omega)$. For the proof it is enough to set $V=W_{0}^{1, p}(\Omega)$ and define the operator $A: V \rightarrow V^{\prime}$ by

$$
\langle A v, w\rangle=\int_{\Omega}|\nabla v|^{p-2} \nabla v \cdot \nabla w d x+\int_{\Omega} \mathcal{A}|H(v)|^{p-2} H(v) w d x \quad \text { for any } w \in V .
$$

Notice that, since $H$ is Lipschitz, $H(v) \in L^{p}(\Omega)$ for any $v \in L^{p}(\Omega)$. Then $A$ is a hemicontinuous strictly monotone coercive operator, and the existence and uniqueness of a weak solution $u$ is standard (see, e.g., [26]).

We will make fundamental use of the following reformulation of a weak solution. Since the limit operator $A: V \rightarrow V^{\prime}$, with $V=W_{0}^{1, p}(\Omega)$, given by (2.5), is hemicontinuous and monotone, we can use the BrezisSibony characterization (see [3, Lemma 1.1] or [26, Chapter 2, Theorem 2.2]), that is, $u \in W_{0}^{1, p}(\Omega)$ is a weak solution of (1.3) if and only if

$$
\int_{\Omega}|\nabla v|^{p-2} \nabla v \cdot \nabla(v-u) d x+\int_{\Omega} \mathcal{B}_{0}|H(v)|^{p-2} H(v)(v-u) d x \geq \int_{\Omega} f(v-u) d x \quad \text { for any } v \in W_{0}^{1, p}(\Omega) .
$$

The main result of this paper is the following convergence result.

Theorem 2.4. Let $n \geq 3,1<p<n, \alpha=\frac{n}{n-p}$ and $\gamma=\alpha(p-1)$. Let $\sigma$ be any maximal monotone graph of $\mathbb{R}^{2}$, with $0 \in \sigma(0)$, and let $f \in L^{p^{\prime}}(\Omega)$. Let $u_{\varepsilon} \in W_{0}^{1, p}\left(\Omega_{\varepsilon}, \partial \Omega\right)$ be the (unique) weak solution of problem (1.1). Then there exists an extension $\tilde{u}_{\varepsilon}$ of $u_{\varepsilon}$ such that $\tilde{u}_{\varepsilon} \rightarrow u$ in $W_{0}^{1, p}(\Omega)$ as $\varepsilon \rightarrow 0$, where $u \in W_{0}^{1, p}(\Omega)$ is the (unique) weak solution of problem (1.3) associated to the function $H$, defined by (1.5).

Remark 2.5. The case $n=2$ can be studied by similar techniques, although some of the computations vary. In particular, the critical value of $\alpha$ does not verify the same formula.

The other key result we will prove in this paper is Theorem 2.9 below, the statement of which requires some preliminary lemmas. The extension $\tilde{u}_{\varepsilon}$ of solutions $u_{\varepsilon}$ can be obtained by applying the methods of [30]. 
Lemma 2.6. Let $\Omega_{\varepsilon}$ be the domain defined above and let $1<p<n, n \geq 3$. Then there exists an extension operator $P_{\varepsilon}: W^{1, p}\left(\Omega_{\varepsilon}\right) \rightarrow W^{1, p}(\Omega)$ such that

$$
\begin{aligned}
\left\|P_{\varepsilon} u\right\|_{W^{1, p}(\Omega)} & \leq C_{1}\|u\|_{W^{1, p}\left(\Omega_{\varepsilon}\right)}, \\
\left\|\nabla\left(P_{\varepsilon} u\right)\right\|_{L_{p}(\Omega)} & \leq C_{2}\|\nabla u\|_{L_{p}\left(\Omega_{\varepsilon}\right)} .
\end{aligned}
$$

Moreover, by applying this extension theorem and the methods introduced in [30], we can prove the following useful estimates.

Lemma 2.7. (i) Let $u \in W_{0}^{1, p}\left(\Omega_{\varepsilon}, \partial \Omega\right), p>1$ and $n \geq 3$. Then there exists positive constant $C$ such that

$$
\|u\|_{L^{p}\left(\Omega_{\varepsilon}\right)} \leq C\|\nabla u\|_{L^{p}\left(\Omega_{\varepsilon}\right)} .
$$

(ii) Let $u \in W^{1, p}\left(Y_{\varepsilon}\right)$ be such that $\int_{Y_{\varepsilon}} u=0$. Then

$$
\|u\|_{L^{p}\left(Y_{\varepsilon}\right)} \leq K_{1} \varepsilon\|\nabla u\|_{L^{p}\left(Y_{\varepsilon}\right)},
$$

where the constant $K_{1}$ is independent of $\varepsilon$.

Thanks to the a priori estimate (2.3) and the properties of the extension operator $P_{\varepsilon}: W_{0}^{1, p}\left(\Omega_{\varepsilon}, \partial \Omega\right) \rightarrow W^{1, p}(\Omega)$, we know that and there exists $u \in W_{0}^{1, p}(\Omega)$ such that

$$
P_{\varepsilon} u_{\varepsilon} \rightarrow u \quad \text { in } W_{0}^{1, p}(\Omega) .
$$

The difficult task is to show that $u \in W_{0}^{1, p}(\Omega)$ is the weak solution of problem (1.3) such as it is ensured in Theorem 2.4.

Motivated by this and (2.6), we will also use the fact that if $u_{\varepsilon} \in W_{0}^{1, p}\left(\Omega_{\varepsilon}, \partial \Omega\right)$ is the weak solution of problem (1.1), then

$$
\int_{\Omega_{\varepsilon}}|\nabla v|^{p-2} \nabla v \cdot \nabla\left(v-u_{\varepsilon}\right) d x+\varepsilon^{-\gamma} \int_{S_{\varepsilon}}\left(\Psi(v)-\Psi\left(u_{\varepsilon}\right)\right) d S \geq \int_{\Omega_{\varepsilon}} f\left(v-u_{\varepsilon}\right) d x
$$

for any test function $v \in W^{1, p}\left(\Omega_{\varepsilon}, \partial \Omega\right)$.

The problematic term, in order to pass to the limit, is the boundary integrals over $S_{\varepsilon}$. Here we will follow a technique of proof introduced by the last author (Shaposhnikova) in collaboration with different co-authors (see, e.g., [28, 34, 37]), which can be applied in different frameworks.

Lemma 2.8. Let $z_{\varepsilon} \in W_{0}^{1, p}(\Omega)$ for some $p>1$, and assume that $z_{\varepsilon} \rightarrow z_{0}$ in $W_{0}^{1, p}(\Omega)$ as $\varepsilon \rightarrow 0$. Then

$$
\left|2^{2(n-1)} \varepsilon \sum_{j \in \Upsilon_{\varepsilon}} \int_{\partial T_{\varepsilon / 4}^{j}} z_{\varepsilon} d S-\omega_{n} \int_{\Omega} z_{0} d x\right| \rightarrow 0 \quad \text { as } \varepsilon \rightarrow 0,
$$

where $\omega_{n}$ is the surface area of the unit sphere in $\mathbb{R}^{n}$.

This lemma (which we remark is independent of $\alpha$ and $\gamma$, see the proof in [37]) is the key point of the homogenization technique in the critical case. It is based in the general idea that if $P_{\varepsilon}^{j}$ is the center of the ball $G_{\varepsilon}^{j}=\left\{x \in Y_{\varepsilon}^{j}:\left|x-P_{\varepsilon}^{j}\right|<a_{\varepsilon}\right\}$ and if $T_{\varepsilon}^{j}$ denotes the ball of radius $\varepsilon / 4$ centered at the point $P_{\varepsilon}^{j}$, then we can get several explicit estimates on the solution $w_{\varepsilon}^{j}(x)$ for $j=1, \ldots, N(\varepsilon)$ of the auxiliary cellular boundary value problem

$$
\begin{cases}\Delta_{p} w_{\varepsilon}^{j}=0, & x \in T_{\varepsilon}^{j} \backslash \overline{G_{\varepsilon}^{j}}, \\ w_{\varepsilon}^{j}=1, & x \in \partial G_{\varepsilon}^{j}, \\ w_{\varepsilon}^{j}=0, & x \in \partial T_{\varepsilon}^{j} .\end{cases}
$$

One of the many remarkable properties of this cellular problem is that its (unique) weak solution, $w_{\varepsilon}^{j}$, is radially symmetric (recall that $G_{0}$ is a ball) and satisfies that $\partial_{v_{p}} w_{\varepsilon}^{j}$ is constant on $\partial T_{\varepsilon}^{j}$ and on $\partial G_{\varepsilon}^{j}$. Due to the divergence theorem,

$$
\int_{G_{\varepsilon}^{j}}\left|\nabla w_{\varepsilon}^{j}\right|^{p-2} \nabla w_{\varepsilon}^{j} \cdot \nabla z d x=\int_{\partial T_{\varepsilon}^{j}} z \partial_{v_{p}} w_{\varepsilon}^{j} d S+\int_{\partial G_{\varepsilon}^{j}} z \partial_{v_{p}} w_{\varepsilon}^{j} d S \quad \text { for any } z \in W^{1, p}\left(T_{\varepsilon}^{j} \backslash \overline{G_{\varepsilon}^{j}}\right) .
$$


Furthermore, we can make explicitly several computations. Hence, we have an explicit way to compare the reaction term on $S_{\varepsilon}$ with an auxiliary term on balls with radius $C \varepsilon$, and Lemma 2.8 becomes very useful.

Another key idea of our proof is to relate a general test function $v \in W_{0}^{1, p}(\Omega)$, used to check the limit characterization (2.6), with some suitable correction $v_{\varepsilon}$, which is a better fitted test function in the microscopic weak formulation (2.7). In fact, by density, it will be enough to do that with a smooth test function $v \in \mathcal{C}_{c}^{\infty}(\Omega)$. We will construct such adaptation among test functions in the form $v_{\varepsilon}=v-h W_{\varepsilon}$, where, for the moment, $h \in W^{1, \infty}(\Omega)$ without any other property, and, which is crucial, $W_{\varepsilon} \in W_{0}^{1, \infty}(\Omega)$ defined as

$$
W_{\varepsilon}= \begin{cases}w_{\varepsilon}^{j}, & x \in T_{\varepsilon}^{j} \backslash \overline{G_{\varepsilon}^{j}}, j=1, \ldots, N(\varepsilon)=\left|\Upsilon_{\varepsilon}\right|, \\ 1, & x \in G_{\varepsilon}, \\ 0, & x \in \mathbb{R}^{n} \backslash \bigcup_{j=1}^{N(\varepsilon)} T_{\varepsilon}^{j},\end{cases}
$$

with $w_{\varepsilon}^{j}$ the solution of the auxiliary cellular boundary value problem (2.8). The following technical result will explain why the function $H$, arising in the limit problem (1.3), was taken in this concrete form (more precisely, so that (2.4) holds), different from the boundary kinetics $\sigma$.

Theorem 2.9. Let $u_{\varepsilon} \in W_{0}^{1, p}\left(\Omega_{\varepsilon}, \partial \Omega\right), 1<p<n$, be a sequence of uniformly bounded norm, and let $v \in \mathcal{C}_{c}^{\infty}(\Omega)$, $h \in W^{1, \infty}(\Omega)$ and $v_{\varepsilon}=v-h W_{\varepsilon}$. Then

$$
\lim _{\varepsilon \rightarrow 0}\left(\int_{\Omega_{\varepsilon}}\left|\nabla v_{\varepsilon}\right|^{p-2} \nabla v_{\varepsilon} \cdot \nabla\left(v_{\varepsilon}-u_{\varepsilon}\right) d x\right)=\lim _{\varepsilon \rightarrow 0}\left(I_{1, \varepsilon}+I_{2, \varepsilon}+I_{3, \varepsilon}\right),
$$

where

$$
\begin{aligned}
& I_{1, \varepsilon}=\int_{\Omega_{\varepsilon}}|\nabla v|^{p-2} \nabla v \cdot \nabla\left(v-u_{\varepsilon}\right) d x, \\
& I_{2, \varepsilon}=-\varepsilon^{-\gamma} \mathcal{B}_{0} \int_{S_{\varepsilon}}|h|^{p-2} h\left(v-h-u_{\varepsilon}\right) d S, \\
& I_{3, \varepsilon}=-A_{\varepsilon} \varepsilon \sum_{j \in Y_{\varepsilon}} \int_{\partial T_{\varepsilon}^{j}}|h|^{p-2} h\left(v-u_{\varepsilon}\right) d S,
\end{aligned}
$$

with $A_{\varepsilon}$ being a bounded sequence, see (5.1). Besides, if $\tilde{u}_{\varepsilon}$ is an extension of $u_{\varepsilon}$ and $\tilde{u}_{\varepsilon} \rightarrow u$ in $W_{0}^{1, p}(\Omega)$, then, for any $v \in W_{0}^{1, p}(\Omega)$,

$$
\lim _{\varepsilon \rightarrow 0} \int_{\Omega_{\varepsilon}}|\nabla v|^{p-2} \nabla v \cdot \nabla\left(v-h W_{\varepsilon}-u_{\varepsilon}\right) d x=\int_{\Omega}|\nabla v|^{p-2} \nabla v \cdot \nabla(v-u) d x .
$$

The aforementioned corrector term in the form $h W_{\varepsilon}$, where $h \in W^{1, \infty}\left(\Omega_{\varepsilon}, \partial \Omega\right)$ will be taken to satisfy the condition $h(x)=H(v(x))$ for a.e. $x \in \Omega$, with $H$ given by (2.4). These conditions rise naturally so that the term $I_{2, \varepsilon}$ above cancels out with the reaction term.

Remark 2.10. In general, it is expected that the convergence $\tilde{u}_{\varepsilon} \rightarrow u$ can be improved to strong convergence by adding a corrector term. In fact, if $\sigma$ is smooth, it is known that $u_{\varepsilon}-H\left(u_{\varepsilon}\right) W_{\varepsilon} \rightarrow u$ strongly in $W_{0}^{1, p}(\Omega)$ (see, e.g., [37]). It is possible to adapt these arguments to the case of some maximal monotone graphs as, for instance, the one given by the Signorini boundary condition (see [12]).

\section{Existence of $u_{\varepsilon}$ and characterization of the function $H$}

Proof of Proposition 2.2. Consider the Banach space $V=W_{0}^{1, p}\left(\Omega_{\varepsilon}, \partial \Omega\right)$. Let $A_{\varepsilon}: V \rightarrow V^{\prime}$ be the operator defined by

$$
\left\langle A_{\varepsilon} v, w\right\rangle=\int_{\Omega_{\varepsilon}}|\nabla v|^{p-2} \nabla v \cdot \nabla w d x \quad \text { for any } w \in W_{0}^{1, p}\left(\Omega_{\varepsilon}, \partial \Omega\right) .
$$


Then $A$ is a hemicontinuous strictly monotone coercive operator [26]. Define $\varphi^{\varepsilon}: W_{0}^{1, p}\left(\Omega_{\varepsilon}, \partial \Omega\right) \rightarrow(-\infty,+\infty$ ] by

$$
\varphi^{\varepsilon}(u)= \begin{cases}\varepsilon^{-\gamma} \int_{S_{\varepsilon}} \Psi\left(\operatorname{tr}_{S_{\varepsilon}}(u)\right) d S & \text { if } \operatorname{tr}_{S_{\varepsilon}}(u(x)) \in D(\Psi) \text { for a.e. } x \in S_{\varepsilon}, \\ +\infty & \text { otherwise. }\end{cases}
$$

It is clear that $\varphi^{\varepsilon}$ is a convex lower semicontinuous function with $\varphi^{\varepsilon} \not \equiv+\infty$. Since $f \in V^{\prime}$, we have that $u_{\varepsilon}$ is a weak solution of problem (1.1) if and only if

$$
\left\langle A_{\varepsilon}\left(u_{\varepsilon}\right)-f, v-u_{\varepsilon}\right\rangle+\varphi^{\varepsilon}(v)-\varphi^{\varepsilon}\left(u_{\varepsilon}\right) \geq 0 \quad \text { for all } v \in V .
$$

Thus, the existence and uniqueness of a weak solution $u_{\varepsilon}$ of problem (1.1) is consequence of [26, Chapter 2, Theorem 8.5].

In order to prove the a priori bound (2.3), let $v \in W_{0}^{1, p}\left(\Omega_{\varepsilon}, \partial \Omega\right)$. Then, we have

$$
\int_{\Omega_{\varepsilon}}\left|\nabla u_{\varepsilon}\right|^{p} d x+\varepsilon^{-\gamma} \int_{S_{\varepsilon}} \Psi\left(u_{\varepsilon}\right) d S \leq \int_{\Omega_{\varepsilon}}\left|\nabla u_{\varepsilon}\right|^{p-2} \nabla u_{\varepsilon} \cdot \nabla v d x+\varepsilon^{-\gamma} \int_{S_{\varepsilon}} \Psi(v) d S-\int_{\Omega_{\varepsilon}} f\left(v-u_{\varepsilon}\right) d x .
$$

Given $\delta \in(0,1)$, we apply Young's inequality, $a b \leq \delta|a|^{p^{\prime}}+C_{\delta}|b|^{p}$, to get

$$
\int_{\Omega_{\varepsilon}}\left|\nabla u_{\varepsilon}\right|^{p-2} \nabla u_{\varepsilon} \cdot \nabla v d x \leq \delta \int_{\Omega_{\varepsilon}}\left|\nabla u_{\varepsilon}\right|^{p} d x+C_{\delta} \int_{\Omega_{\varepsilon}}|\nabla v|^{p} d x .
$$

Therefore, since $\Psi \geq 0$, taking $v=0$ and applying Hölder's and Poincaré's inequalities, we have

$$
(1-\delta)\left\|\nabla u_{\varepsilon}\right\|_{L^{p}\left(\Omega_{\varepsilon}\right)}^{p}+\varepsilon^{-\gamma}\left\|\Psi\left(u_{\varepsilon}\right)\right\|_{L^{1}\left(S_{\varepsilon}\right)} \leq \int_{\Omega_{\varepsilon}} f u_{\varepsilon} d x \leq C\|f\|_{L^{p^{\prime}}(\Omega)}\left\|\nabla u_{\varepsilon}\right\|_{L^{p}\left(\Omega_{\varepsilon}\right)},
$$

which leads to the result.

Proof of Lemma 2.3. Let $\Theta_{n, p}(s)=\mathcal{B}_{0}|s|^{p-2} s$ for $s \in \mathbb{R}$. Since $\sigma^{-1}$ is also a maximal monotone graph of $\mathbb{R}^{2}$, for any $p>1$ and $\mathcal{B}_{0}>0$, the graph $\sigma^{-1} \circ \Theta_{n, p}$ is also a maximal monotone graph of $\mathbb{R}^{2}$. Indeed, let $D\left(\sigma^{-1}\right)=[a, b]$ for some $-\infty \leq a<b \leq+\infty$, and let $\left(\sigma^{-1}\right)^{0}$ be the principal section (i.e., the nondecreasing function) of the graph $\sigma^{-1}$. This means that

$$
\left(\sigma^{-1}\right)^{0}(r)=\inf \sigma^{-1}(r), \quad r \in[a, b] .
$$

Then, since $\Theta_{n, p}$ is strictly increasing, $\sigma^{-1} \circ \Theta_{n, p}$ is a monotone graph,

$$
D\left(\sigma^{-1} \circ \Theta_{n, p}\right)=\left[\Theta_{n, p}^{-1}(a), \Theta_{n, p}^{-1}(b)\right] \text { and }\left(\sigma^{-1} \circ \Theta_{n, p}\right)^{0}=\left(\sigma^{-1}\right)^{0} \circ \Theta_{n, p} .
$$

In particular, if $\sigma^{-1}$ is multivalued in some point $c \in(a, b)$, then $\sigma^{-1} \circ \Theta_{n, p}(c)$ is the full interval

$$
\sigma^{-1} \circ \Theta_{n, p}(c)=\left[\left(\sigma^{-1}\right)^{0}\left(\Theta_{n, p}(c)^{-}\right),\left(\sigma^{-1}\right)^{0}\left(\Theta_{n, p}(c)^{+}\right)\right],
$$

and this implies that $\sigma^{-1} \circ \Theta_{n, p}$ is a maximal monotone graph of $\mathbb{R}^{2}$ (see [2, Example 2.8.1]).

Now, since $\sigma^{-1} \circ \Theta_{n, p}$ is also a maximal monotone graph of $\mathbb{R}^{2}$, we know that $\left(I+\sigma^{-1} \circ \Theta_{n, p}\right)$ is an injective application such that $R\left(I+\sigma^{-1} \circ \Theta_{n, p}\right)=\mathbb{R}$ (see [2]). Thus, if $H$ is defined by (1.5), then $H$ is a nonexpansion on $\mathbb{R}$ (see [2, Proposition 2.2]). Hence,

$$
\left(I+\sigma^{-1} \circ \Theta_{n, p}\right)(H(r))=r
$$

for any $r \in \mathbb{R}$ and, in consequence,

$$
H(r)+\sigma^{-1} \circ \Theta_{n, p}(H(r))=r
$$

In other words,

$$
\sigma^{-1} \circ \Theta_{n, p}(H(r))=r-H(r)
$$


This implies that $r-H(r) \in D(\sigma)$ for any $r \in \mathbb{R}$ and that $\Theta_{n, p}(H(r)) \in \sigma(r-H(r))$ for any $r \in \mathbb{R}$, which proves that $H(r)$ satisfies relation (2.4). Moreover, from the definition of $H$, it is obvious that $H$ is nondecreasing (in fact if $\sigma$ is strictly increasing, then $H$ is also a strictly increasing function).

On the other hand, such function $H(r)$ is the unique function satisfying relation (2.4), since applying the inverse graph

$$
\sigma^{-1} \circ \Theta_{n, p} \circ H \supset(I-H)
$$

implies that $\left(I+\sigma^{-1} \circ \Theta_{n, p}\right) \circ H=I$, and so, necessarily, $H=\left(I+\sigma^{-1} \circ \Theta_{n, p}\right)^{-1}$. Of course, from the implicit formula, $H$ is strictly increasing.

\section{Proof of Theorem 2.4}

Since $G_{0}$ is ball, it is easy to see that

$$
w_{\varepsilon}^{j}(x)=\frac{\left|x-P_{\varepsilon}^{j}\right|^{-\frac{n-p}{p-1}}-(\varepsilon / 4)^{-\frac{n-p}{p-1}}}{\left(C_{0} \varepsilon^{\alpha}\right)^{-\frac{n-p}{p-1}}-(\varepsilon / 4)^{-\frac{n-p}{p-1}}}, \quad x \in T_{\varepsilon}^{j}, \backslash \overline{G_{\varepsilon}^{j}},
$$

is the unique solution of (2.8).

Lemma 4.1. If $W_{\varepsilon}$ is defined by (2.9), then the following estimate holds:

$$
\int_{\Omega_{\varepsilon}}\left|\nabla W_{\varepsilon}\right|^{q} d x \leq K \varepsilon^{\frac{n(p-q)}{n-p}} \quad \text { for any } 1 \leq q \leq p .
$$

In particular,

$$
W_{\varepsilon} \rightarrow 0 \quad \text { in } W_{0}^{1, p}(\Omega) \text { as } \varepsilon \rightarrow 0 .
$$

Proof. Estimate (4.2) is an explicit computation. For $q=p$, we obtain from it that, up to a subsequence, there exists $W_{0} \in W_{0}^{1, p}(\Omega)$ such that $W_{\varepsilon} \rightarrow W_{0}$ in $W_{0}^{1, p}(\Omega)$. For $q<p$, we have that $W_{\varepsilon} \rightarrow 0$ in $W_{0}^{1, q}(\Omega)$, hence $W_{0}=0$.

Proof of Theorem 2.4. Let $v \in \mathcal{C}_{c}^{\infty}(\Omega)$ and $h=H(v)$, with $H: \mathbb{R} \rightarrow \mathbb{R}$ given by (1.5). Then $h \in W^{1, \infty}(\Omega)$. Let $v_{\varepsilon}=v-h W_{\varepsilon} \in W_{0}^{1, p}\left(\Omega_{\varepsilon}, \partial \Omega\right)$, with $W_{\varepsilon} \in W_{0}^{1, \infty}(\Omega)$ defined by (2.9). Due to (2.7), we know that $u_{\varepsilon}$ satisfies the inequality

$$
\int_{\Omega_{\varepsilon}}\left|\nabla v_{\varepsilon}\right|^{p-2} \nabla v_{\varepsilon} \cdot \nabla\left(v_{\varepsilon}-u_{\varepsilon}\right) d x+\varepsilon^{-\gamma} \int_{S_{\varepsilon}}\left(\Psi\left(v_{\varepsilon}\right)-\Psi\left(u_{\varepsilon}\right)\right) d S \geq \int_{\Omega_{\varepsilon}} f\left(v_{\varepsilon}-u_{\varepsilon}\right) d x
$$

Since $W_{\varepsilon} \rightarrow 0$ in $L^{p}(\Omega)$ (due to the compact inclusion), by Theorem 2.9, we can deduce that

$$
\lim _{\varepsilon \rightarrow 0}\left[I_{1, \varepsilon}+I_{2, \varepsilon}+I_{3, \varepsilon}+\varepsilon^{-\gamma} \int_{S_{\varepsilon}}\left(\Psi\left(v_{\varepsilon}\right)-\Psi\left(u_{\varepsilon}\right)\right) d S\right] \geq \lim _{\varepsilon \rightarrow 0} \int_{\Omega_{\varepsilon}} f\left(v_{\varepsilon}-u_{\varepsilon}\right) d x=\int_{\Omega} f(v-u) d x .
$$

Since $H: \mathbb{R} \rightarrow \mathbb{R}$ satisfies (2.4), by applying that if $\xi \in \partial \Psi\left(s_{0}\right)=\sigma\left(s_{0}\right)$, then $\Psi(s)-\Psi\left(s_{0}\right) \geq \xi\left(s-s_{0}\right)$, we can write

$$
I_{2, \varepsilon}+\varepsilon^{-\gamma} \int_{S_{\varepsilon}}\left(\Psi\left(v_{\varepsilon}\right)-\Psi\left(u_{\varepsilon}\right)\right) d S=\varepsilon^{-\gamma} \int_{S_{\varepsilon}}\left[\Psi(v-H(v))-\Psi\left(u_{\varepsilon}\right)-\mathcal{B}_{0}|H(v)|^{p-2} H(v)\left(v-H(v)-u_{\varepsilon}\right)\right] d S \leq 0,
$$

since $\mathcal{B}_{0}|H(v(x))|^{p-2} H(v(x)) \in \sigma(v(x)-H(v(x)))$ for any $x \in \bar{\Omega}$. We can pass also to the limit in (2.10) and (2.11) to get that

$$
\int_{\Omega}|\nabla v|^{p-2} \nabla v \nabla(v-u) d x+\int_{\Omega} \mathcal{B}_{0}|H(v)|^{p-2} H(v)(v-u) d x \geq \int_{\Omega} f(v-u) d x,
$$

and since $v \in \mathcal{C}_{c}^{\infty}(\Omega)$ is arbitrary, by density, this also holds for every $v \in W_{0}^{1, p}(\Omega)$. Hence, we get that $u$ is the unique weak solution of (1.3). 


\section{Proof of Theorem 2.9}

The proof of Theorem 2.9 for $p=2$ can be found in [36], and for $2<p<n$ in [34]. Here we will complete the proof for $1<p<2$. We need some auxiliary results.

Lemma 5.1 ([12]). Let $1<p<2$. Then there exists positive constant $C=C(p)$ such that the inequality

$$
|| \mathbf{a}-\left.\mathbf{b}\right|^{p-2}(\mathbf{a}-\mathbf{b})-\left(|\mathbf{a}|^{p-2} \mathbf{a}-|\mathbf{b}|^{p-2} \mathbf{b}\right) \mid \leq C(|\mathbf{a}||\mathbf{b}|)^{\frac{p-1}{2}}
$$

is valid for all $\mathbf{a}, \mathbf{b} \in \mathbb{R}^{n}$.

By using this result, we prove the following lemma.

Lemma 5.2. Let $1<p<2, n \geq 3, v \in W_{0}^{1, \infty}(\Omega)$ and $\varphi \in W_{0}^{1, p}(\Omega)$. Let $\eta_{\varepsilon} \in W^{1, p}(\Omega)$ be such that $\left\|\nabla \eta_{\varepsilon}\right\|_{L^{q}(\Omega)} \rightarrow 0$ for some $q \in[1, p)$ as $\varepsilon \rightarrow 0$. Then

$$
\lim _{\varepsilon \rightarrow 0}\left(\int_{\Omega_{\varepsilon}}\left|\nabla\left(v-\eta_{\varepsilon}\right)\right|^{p-2} \nabla\left(v-\eta_{\varepsilon}\right) \cdot \nabla \varphi d x\right)=\lim _{\varepsilon \rightarrow 0}\left(\int_{\Omega_{\varepsilon}}|\nabla v|^{p-2} \nabla v \cdot \nabla \varphi d x-\int_{\Omega_{\varepsilon}}\left|\nabla \eta_{\varepsilon}\right|^{p-2} \nabla \eta_{\varepsilon} \cdot \nabla \varphi d x\right) .
$$

Proof. By Lemma 5.1, by applying Hölder's inequality, we have

since $1<\frac{p+1}{2}<p$. This proves the result.

$$
\begin{aligned}
\left.\left|\int_{\Omega_{\varepsilon}}\right| \nabla\left(v-\eta_{\varepsilon}\right)\right|^{p-2} \nabla\left(v-\eta_{\varepsilon}\right) \cdot \nabla \varphi d x-\left(\left.\nabla v\right|^{p-2} \nabla v-\left|\nabla \eta_{\varepsilon}\right|^{p-2} \nabla \eta_{\varepsilon}\right) \cdot \nabla \varphi d x \mid \\
\quad \leq C \int_{\Omega_{\varepsilon}}|\nabla v|^{\frac{p-1}{2}}\left|\nabla \eta_{\varepsilon}\right|^{\frac{p-1}{2}}|\nabla \varphi| d x \\
\quad \leq K\|\nabla v\|_{\infty}^{\frac{p-1}{2}}\left\|\nabla \eta_{\varepsilon}\right\|_{L^{\frac{p-1}{2}}\left(\Omega_{\varepsilon}\right)}^{\frac{p+1}{2}}\|\nabla \varphi\|_{L^{\frac{p+1}{2}}\left(\Omega_{\varepsilon}\right)},
\end{aligned}
$$

We have all the tools we need for the proof of Theorem 2.9.

Proof of Theorem 2.9. As said before, it is enough to consider the case $p \in(1,2)$. Applying Lemma 5.2, we obtain

$$
\lim _{\varepsilon \rightarrow 0}\left(\int_{\Omega_{\varepsilon}}\left|\nabla v_{\varepsilon}\right|^{p-2} \nabla v_{\varepsilon} \cdot \nabla\left(v_{\varepsilon}-u_{\varepsilon}\right) d x\right)=\lim _{\varepsilon \rightarrow 0}\left(J_{1, \varepsilon}+J_{2, \varepsilon}\right),
$$

where

$$
\begin{aligned}
& J_{1, \varepsilon}=\int_{\Omega_{\varepsilon}}|\nabla v|^{p-2} \nabla v \cdot \nabla\left(v-h W_{\varepsilon}-u_{\varepsilon}\right) d x, \\
& J_{2, \varepsilon}=\int_{\Omega_{\varepsilon}}\left|\nabla\left(h W_{\varepsilon}\right)\right|^{p-2} \nabla\left(h W_{\varepsilon}\right) \cdot \nabla\left(v-h W_{\varepsilon}-u_{\varepsilon}\right) d x .
\end{aligned}
$$

Moreover,

$$
\lim _{\varepsilon \rightarrow 0} J_{1, \varepsilon}=\lim _{\varepsilon \rightarrow 0}\left(I_{1, \varepsilon}+\int_{\Omega_{\varepsilon}}|\nabla v|^{p-2} \nabla v \cdot \nabla\left(h W_{\varepsilon}\right) d x\right)=\lim _{\varepsilon \rightarrow 0} I_{1, \varepsilon}
$$

On the other hand,

$$
\begin{aligned}
\lim _{\varepsilon \rightarrow 0} J_{2, \varepsilon} & =\lim _{\varepsilon \rightarrow 0}\left(\int_{\Omega_{\varepsilon}}\left|\nabla W_{\varepsilon}\right|^{p-2} \nabla W_{\varepsilon} \cdot \nabla\left(v-h W_{\varepsilon}-u_{\varepsilon}\right) d x\right) \\
& =\lim _{\varepsilon \rightarrow 0}\left(\sum_{j \in Y_{\varepsilon}} \int_{\partial T_{\varepsilon}^{j}}\left|\nabla w_{\varepsilon}^{j}\right|^{p-2} \partial_{\nu} w_{\varepsilon}^{j}|h|^{p-2} h\left(v-u_{\varepsilon}\right) d S+\sum_{j \in Y_{\varepsilon}} \int_{\partial G_{\varepsilon}^{j}}\left|\nabla w_{\varepsilon}^{j}\right|^{p-2} \partial_{\nu} w_{\varepsilon}^{j}|h|^{p-2} h\left(v-h-u_{\varepsilon}\right) d S\right),
\end{aligned}
$$

where $\partial_{\nu} g$ is the usual normal derivative of $g$. Using (4.1), we get

$$
\begin{aligned}
& \left.\partial_{\nu} w_{\varepsilon}^{j}\right|_{\partial T_{\varepsilon}^{j}}=\left.\frac{d}{d r} w_{\varepsilon}^{j}\right|_{r=\varepsilon / 4}=-\frac{(n-p) 2^{\frac{2 n-2}{p-1}} C_{0}^{\frac{n-p}{p-1}} \varepsilon^{\frac{1}{p-1}}}{(p-1)\left(1-\left(C_{0} \varepsilon^{\alpha}\right)^{\frac{n-p}{p-1}} \varepsilon^{-\frac{n-p}{p-1}} 2^{\frac{2 n-2 p}{p-1}}\right)}, \\
& \left.\partial_{\nu} w_{\varepsilon}^{j}\right|_{\partial G_{\varepsilon}^{j}}=-\left.\frac{d}{d r} w_{\varepsilon}^{j}\right|_{r=a_{\varepsilon}}=\frac{(n-p) \varepsilon^{\frac{-n}{n-p}}}{(p-1) C_{0}\left(1-\left(C_{0} \varepsilon^{\alpha}\right)^{\frac{n-p}{p-1}} \varepsilon^{-\frac{n-p}{p-1}} 2^{\frac{2 n-2 p}{p-1}}\right)} .
\end{aligned}
$$


Therefore,

$$
\lim _{\varepsilon \rightarrow 0} J_{2, \varepsilon}=\lim _{\varepsilon \rightarrow 0}\left(A_{\varepsilon} \varepsilon \sum_{j \in Y_{\varepsilon}} \int_{\partial T_{\varepsilon}^{j}}|h|^{p-2} h\left(v-u_{\varepsilon}\right) d s-\varepsilon^{-\gamma} \int_{S_{\varepsilon}}\left(\left(\frac{n-p}{p-1}\right)^{p-1} C_{0}^{1-p}|h|^{p-2} h\right)\left(v-h-u_{\varepsilon}\right) d s-Q_{\varepsilon}\right),
$$

where

$$
A_{\varepsilon}=\left(\frac{n-p}{p-1}\right)^{p-1} \frac{2^{2 n-2} C_{0}^{n-p}}{\left(1-\left(C_{0} \varepsilon^{\alpha}\right)^{\frac{n-p}{p-1}} \varepsilon^{-\frac{n-p}{p-1}} 2^{\frac{2 n-2 p}{p-1}}\right)^{p-1}}
$$

and

$$
Q_{\varepsilon}=\frac{1-\left(1-a_{\varepsilon}^{\frac{n-p}{p-1}} \varepsilon^{\frac{p-n}{p-1}} 2^{\frac{2 n-2 p}{p-1}}\right)^{p-1}}{C_{0}^{p-1}\left(1-a_{\varepsilon}^{\frac{n-p}{p-1}} \varepsilon^{\frac{p-n}{p-1}} 2^{\frac{2 n-2 p}{p-1}}\right)^{p-1}}\left(\frac{n-p}{p-1}\right)^{p-1} \varepsilon^{-\gamma} \int_{S_{\varepsilon}}|h|^{p-2} h\left(v-h-u_{\varepsilon}\right) d S .
$$

It is an easy (but tedious) task to check that

$$
\lim _{\varepsilon \rightarrow 0} Q_{\varepsilon}=0
$$

which concludes the proof.

\section{Noncritical case and pointwise comparison of homogenized solutions with the critical case}

For $A \subset \mathbb{R}^{m}$, let $\mathcal{C}(A)$ denote the space of continuous functions on $A$.

Theorem 6.1. Let $n \geq 3, p \in[2, n), 1<\alpha<\frac{n}{n-p}, f \in L^{\infty}(\Omega)$ and $r \in \mathcal{C}(\bar{\Omega})$. Let also $\sigma \in \mathcal{C}(\mathbb{R})$ be nondecreasing such that $\sigma(0)=0$ and let $u_{\varepsilon}$ be the solution of

$$
\begin{cases}-\Delta_{p} u_{\varepsilon}=f, & x \in \Omega_{\varepsilon} \\ \partial_{v_{p}} u_{\varepsilon}+\varepsilon^{-\gamma} \sigma\left(u_{\varepsilon}\right)=\varepsilon^{-\gamma} r, & x \in S_{\varepsilon} \\ u_{\varepsilon}=0, & x \in \partial \Omega .\end{cases}
$$

Then $\tilde{u}_{\varepsilon} \rightarrow u_{\text {non-crit }}$ in $W_{0}^{1, p}(\Omega)$, where $u_{\text {non-crit }}$ is the solution of

$$
\begin{cases}-\Delta_{p} u+\hat{\mathcal{A}} \sigma(u)=f+\hat{\mathcal{A}} r & \text { in } \Omega \\ u=0 & \text { on } \partial \Omega\end{cases}
$$

with $\hat{\mathcal{A}}=C_{0}^{n-1}\left|\partial G_{0}\right|$.

Proof. Assume first that

$$
0<k_{1} \leq \sigma^{\prime} \leq k_{2}
$$

Then the result holds by [34, Theorem 3].

Applying the estimates in [29], we check that $\left(P_{\varepsilon} u_{\varepsilon}\right)$ is bounded in $W_{0}^{1, p}(\Omega)$, hence there exists a limit $\hat{u}$ such that, up to a subsequence, $P_{\varepsilon} u_{\varepsilon} \rightarrow \hat{u}$ strongly in $L^{p}(\Omega)$ and weakly in $W_{0}^{1, p}(\Omega)$.

Let $M$ be such that $\left\|u_{\varepsilon}\right\|_{L^{\infty}\left(\Omega_{\varepsilon}\right)} \leq M$ (see [8]). Let $\sigma_{\delta}$ be a sequence such that $0<k_{1, \delta} \leq \sigma_{\delta}^{\prime} \leq k_{2, \delta}$ and $\sigma_{\delta} \rightarrow \sigma$ in $\mathcal{C}([-M, M])$ as $\delta \rightarrow 0$. Let $u_{\varepsilon, \delta}$ be the solution of (6.1) with $\sigma_{\delta}$. We can check, again from estimates in [29], that

$$
\left\|u_{\varepsilon}-u_{\varepsilon, \delta}\right\|_{L^{p}\left(\Omega_{\varepsilon}\right)} \leq C\left\|\sigma-\sigma_{\delta}\right\| \mathcal{e}([-M, M]) .
$$

Passing to the limit as $\varepsilon \rightarrow 0$, indicating that $P_{\varepsilon} u_{\varepsilon, \delta} \rightarrow u_{\delta}$ in $W_{0}^{1, p}(\Omega)$, where $u_{\delta}$ is the solution of (6.2) with $\sigma_{\delta}$, we have that

$$
\left\|\hat{u}-u_{\delta}\right\|_{L^{p}(\Omega)} \leq C\left\|\sigma-\sigma_{\delta}\right\|_{\mathcal{e}([-M, M])} .
$$

It is easy to check that $u_{\delta} \rightarrow u$ in $L^{p}(\Omega)$, where $u$ is the solution of the problem with $\sigma$. Therefore, $P_{\varepsilon} u_{\varepsilon} \rightarrow u$ in $L^{p}(\Omega)$ as $\varepsilon \rightarrow 0$ and $u=\hat{u}$. 
Theorem 6.2. Let $n \geq 3, p \in[2, n), \alpha=\frac{n}{n-p}, f \in L^{\infty}(\Omega)$ and $r \in \mathcal{C}(\bar{\Omega})$. Let also $\sigma \in \mathcal{C}(\mathbb{R})$ be nondecreasing such that $\sigma(0)=0$ and let $u_{\varepsilon}$ be the solution of (6.1). Then $\tilde{u}_{\varepsilon} \rightarrow u_{\text {crit }}$ in $W_{0}^{1, p}(\Omega)$, where $u_{\text {crit }}$ is the solution of

$$
\begin{cases}-\Delta_{p} u+\mathcal{A}|H(x, u)|^{p-2} H(x, u)=f & \text { in } \Omega, \\ u=0 & \text { on } \partial \Omega,\end{cases}
$$

and $H$ is the solution of

$$
\mathcal{B}_{0}|H(x, s)|^{p-2} H(x, s)=\sigma(s-H(x, s))-r(x) \quad \text { a.e. in } \Omega \text {. }
$$

Sketch of proof. We can apply the same reasoning as before and the fact that $H_{\delta} \rightarrow H$, in the sense of maximal monotone graphs, as $\sigma_{\delta} \rightarrow \sigma$ in $\mathcal{C}([-M, M])$.

Theorem 6.3. Assume the conditions of the two previous theorems, $f=0$ and $r(x) \equiv g(1)=1$. Then, we have that $u_{\text {crit }} \leq u_{\text {non-crit }}$.

Proof. The condition on $f$ and $r$ guarantee that $0 \leq u \leq 1$ in both cases. It is easy to check that $H$ is increasing, and $H(s) \leq 0$ for $s \in[0,1]$. It is easy to establish the following inequality on the zero order terms:

$$
\mathcal{B}_{0}|H(s)|^{p-2} H(s) \geq \hat{\mathcal{A}}(\sigma(s)-g(1)) .
$$

Therefore, applying the comparison principle (see, e.g., [8]), we have the result.

Funding: The research of the first two authors was partially supported by the project ref. MTM2014-57113-P of the DGISPI (Spain) and as members of the Research Group MOMAT (Ref. 910480) of the UCM . The research of D. Gómez-Castro was supported by an FPU Grant from the Ministerio de Educación, Cultura y Deporte (Spain) (Ref. FPU14/03702).

\section{References}

[1] H. Brézis, Monotonicity methods in Hilbert spaces and some applications to nonlinear partial differential equations, in: Contributions to Nonlinear Functional Analysis, Academic Press, New York (1971), 101-156.

[2] H. Brézis, Operateurs Maximaux Monotones et Semi-groupes de Contractions dans les Espaces de Hilbert, North-Holland, Amsterdam, 1973.

[3] H. Brézis and M. Sibony, Méthodes d'approximation et d'itération pour les opérateurs monotones, Arch. Ration. Mech. Anal. 28 (1968), no. 1, 59-82.

[4] D. Cioranescu and F. Murat, A strange term coming from nowhere, in: Topics in the Mathematical Modelling of Composite Materials, Progr. Nonlinear Differential Equations Appl. 31, Birkhäuser, Boston (1997), 45-93.

[5] C. Conca, J. I. Díaz, A. Liñán and C. Timofte, Homogenization in chemical reactive flows, Electron. J. Differential Equations (2004) 2004, Paper No. 40.

[6] C. Conca and P. Donato, Nonhomogeneous Neumann problems in domains with small holes, RAIRO Modél. Math. Anal. Numér. 22 (1988), no. 4, 561-607.

[7] G. Dal Maso and I. V. Skrypnik, A monotonicity approach to nonlinear Dirichlet problems in perforated domains, Adv. Math. Sci. Appl. 11 (2001), no. 2, 721-751.

[8] J. I. Díaz, Nonlinear Partial Differential Equations and Free Boundaries. Vol. I: Elliptic Equations, Res. Notes Math. 106, Pitman, London, 1985.

[9] J. I. Díaz, Two problems in homogenization of porous media, Extracta Math. 14 (1999), no. 2, 141-155.

[10] J. I. Díaz and D. Gómez-Castro, A mathematical proof in nanocatalysis: Better homogenized results in the diffusion of a chemical reactant through critically small reactive particles, in: Progress in Industrial Mathematics at ECMI 2016, Springer, Cham (2017), to appear.

[11] J. I. Díaz, D. Gómez-Castro, A. V. Podol'skii and T. A. Shaposhnikova, Homogenization of the $p$-Laplace operator with nonlinear boundary condition on critical size particles: Identifying the strange terms for some non smooth and multivalued operators, Dokl. Math. 94 (2016), no. 1, 387-392.

[12] J. I. Díaz, D. Gómez-Castro, A. V. Podol'skii and T. A. Shaposhnikova, Homogenization of variational inequalities of Signorini type for the $p$-Laplacian in perforated domains when $p \in(1,2)$, Dokl. Math. 95 (2017), no. 2, 151-156. 
[13] J. I. Díaz, D. Gómez-Castro, A. V. Podol'skii and T. A. Shaposhnikova, Non existence of critical scales in the homogenization of the problem with $p$-Laplace diffusion and nonlinear reaction in the boundary of periodically distributed particles in $n$-dimensional domains when $p \geq n$, Rev. R. Acad. Cienc. Exactas Fís. Nat. Ser. A Math. RACSAM (2017), DOI 10.1007/s13398-017-0381-z.

[14] J. I. Díaz, D. Gómez-Castro, A. V. Podol'skii and T. A. Shaposhnikova, On the asymptotic limit of the effectiveness of reaction-diffusion equations in periodically structured media, J. Math. Anal. Appl. 455 (2017), 1597-1613.

[15] J. I. Díaz, D. Gómez-Castro, T. A. Shaposhnikova and M. N. Zubova, Change of homogenized absorption term in diffusion processes with reaction on the boundary of periodically distributed asymmetric particles of critical size, Electron. J. Differential Equations 2017 (2017), no. 178, 1-25.

[16] D. Gómez, M. Lobo, M. E. Pérez, A. Podolskii and T. A. Shaposhnikova, Unilateral problems for the $p$-Laplace operator in perforated media involving large parameters, ESAIM Control Optim. Calc. Var. (2017), DOI 10.1051/cocv/2017026.

[17] D. Gómez, M. E. Pérez, A. V. Podol'skii and T. A. Shaposhnikova, Homogenization of the $p$-Laplacian in a perforated domain with nonlinear restrictions given on the boundary of the perforations: The critical case, Dokl. Akad. Nauk 463 (2015), no. 3, 259-264.

[18] M. Goncharenko, The asymptotic behaviour of the third boundary-value problem solutions in domains with fine-grained boundaries, in: Homogenization and Applications to Material Sciences (Nice 1995), GAKUTO Internat. Ser. Math. Sci. Appl. 9, Gakkōtosho, Tokyo (1995), 203-213.

[19] E. J. Hruslov, The Dirichlet problem in a region with a random boundary, Vestnik Harkov. Gos. Univ. 53 (1970), $14-37$.

[20] E. J. Hruslov, The first boundary value problem in domains with a complicated boundary for higher order equations, Math. USSR Sb. 32 (1977), no. 4, 535.

[21] E. J. Hruslov, Asymptotic behavior of the solutions of the second boundary value problem in the case of the refinement of the boundary of the domain, Mat. Sb. 106(148) (1978), no. 4, 604-621.

[22] W. Jäger, M. Neuss-Radu and T. A. Shaposhnikova, Homogenization of a variational inequality for the Laplace operator with nonlinear restriction for the flux on the interior boundary of a perforated domain, Nonlinear Anal. Real World Appl. 15 (2014), 367-380.

[23] V. V. Jikov, S. M. Kozlov and O. A. Oleǐnik, Homogenization of Differential Operators and Integral Functionals, Springer, Berlin, 1994.

[24] S. Kaizu, The Poisson equation with semilinear boundary conditions in domains with many tiny holes, J. Fac. Sci. Univ. Tokyo Sect. I A Math. 36 (1989), no. 1, 43-86.

[25] S. Kaizu, The Poisson equation with nonautonomous semilinear boundary conditions in domains with many time holes, SIAM J. Math. Anal. 22 (1991), no. 5, 1222-1245.

[26] J.-L. Lions, Quelques Méthodes de Résolution des Problèmes aux Limites Non Linéaires, Dunod, Paris, 1969.

[27] V. A. Marčenko and E. J. Hruslov, Boundary-value problems with fine-grained boundary, Mat. Sb. (N.S.) 65 (107) (1964), 458-472.

[28] O. A. Oleinik and T. A. Shaposhnikova, On the homogenization of the Poisson equation in partially perforated domains with arbitrary density of cavities and mixed type conditions on their boundary, Atti Accad. Naz. Lincei Cl. Sci. Fis. Mat. Natur. Rend. Lincei Mat. Appl. (9) 7 (1996), no. 3, 129-146.

[29] A. V. Podol'skii, Homogenization limit for the boundary value problem with the $p$-Laplace operator and a nonlinear third boundary condition on the boundary of the holes in a perforated domain, Dokl. Math. 82 (2010), no. 3, 942-945.

[30] A. V. Podol'skii, Solution continuation and homogenization of a boundary value problem for the $p$-Laplacian in a perforated domain with a nonlinear third boundary condition on the boundary of holes, Dokl. Math. 91 (2015), no. 1, 30-34.

[31] A. V. Podol'skii and T. A. Shaposhnikova, Homogenization for the $p$-Laplacian in an $n$-dimensional domain perforated by very thin cavities with a nonlinear boundary condition on their boundary in the case $p=n$, Dokl. Math. 92 (2015), no. 1, 464-470.

[32] A. V. Podol'skii and T. A. Shaposhnikova, Homogenization of the $p$-Laplacian in an $n$-dimensional domain perforated by shallow cavities with a nonlinear boundary condition on their boundary in the case when $p=n$ (in Russian), Dokl. Akad. Nauk 463 (2015), no. 4, 395-401.

[33] S. Schimpf, M. Lucas, C. Mohr, U. Rodemerck, A. Brückner, J. Radnik, H. Hofmeister and P. Claus, Supported gold nanoparticles: In-depth catalyst characterization and application in hydrogenation and oxidation reactions, Catalysis Today 72 (2002), no. 1, 63-78.

[34] T. Shaposhnikova and A. Podolskiy, Homogenization limit for the boundary value problem with the $p$-Laplace operator and a nonlinear third boundary condition on the boundary of the holes in a perforated domain, Funct. Differ. Equ. 19 (2012), no. 3-4, 351-370.

[35] J. M. Vega and A. Liñán, Isothermal $n$th order reaction in catalytic pellets: Effect of external mass transfer resistance, Chem. Eng. Sci. 34 (1979), no. 11, 1319-1322.

[36] M. N. Zubova and T. A. Shaposhnikova, Homogenization of boundary value problems for the Laplace operator in perforated domains with a nonlinear boundary condition of the third type on the boundary of holes, Sovrem. Mat. Fundam. Napravl. 39 (2011), 173-184. 
[37] M. N. Zubova and T. A. Shaposhnikova, Averaging of boundary-value problems for the Laplace operator in perforated domains with a nonlinear boundary condition of the third type on the boundary of cavities, J. Math. Sci. 190 (2013), 181-193. 